\title{
INSIGHTS
}

\section{Family reflections: pediatric organ transplant}

\author{
Jen $\mathrm{Lau}^{1}$ \\ Pediatric Research (2020) 87:967-968; https://doi.org/10.1038/s41390-019-0678-z
}

My son Nathan was born with a rare liver disease, called biliary atresia (BA). BA is when the bile ducts are blocked, not allowing normal bile flow and causing damage to the liver, which is life threatening without treatment. Nathan was diagnosed with BA at age 6 weeks at Ann and Robert H. Lurie Children's Hospital of Chicago, now a very familiar place to us. He went through his first procedure called a Kasai, which is a surgical treatment to try and help the bile drain and not cause further damage to the liver. Three months following the Kasai surgery, it was clear the procedure was deemed unsuccessful, the liver started to deteriorate, and a liver transplant would be the only treatment to save his life. At the time of his transplant evaluation, we learned Nathan was very fortunate that he was a good candidate to receive a living donor liver transplant, and his team of doctors informed us that this would be his better outcome in surviving his liver disease. We started identifying friends and family who might be suitable donors, and in that process, we also found complete strangers who were willing to help save Nathan's life. The living donor process can be lengthy. The medical team for the donor looks at blood type, medical history, and the psychological and social support. The possible donor will also have imaging done, and in some cases, a liver biopsy is performed. We had a couple of family and friends go through this process only to find out that they were not a match for Nate. Then, while Nathan was admitted to Lurie Children's Hospital to treat an episode of cholangitis (a serious infection of the liver's bile ducts), I received a phone call that I will never forget. A dear friend of mine called to tell me that she learned she was a match for Nate and that she accepted to be his living donor. I think this was the first time that I had exhaled in months. Tears of so many mixed emotions started to fill my eyes. She was our hero, Nathan's guardian angel. At 10 months old, Nathan and my dear friend went into surgery, he received his gift of life, she recovered fully, and to this day, they both have never looked back.

Today, the posttransplant care Nate receives is a multidisciplinary approach. This includes Pediatric Hepatology, Nutrition, Social Work, and any other departments deemed necessary to providing the best care for my child (e.g., General Pediatrics, Allergy, Dermatology, Dental, etc). Nathan goes to clinic every 6 months right now, has routine laboratories, and sometimes imaging to make sure his transplanted liver is functioning properly. Part of the transplant recovery or treatment is being on immune suppression medications. These medications allow Nathan's body to continue to keep the graft he received, and he will be on these for the rest of his life. His transplant team works very closely with other disciplines to help manage his general care so he can continue to have the best quality of life possible post liver transplant.
As a parent, nothing can prepare you for something of this magnitude. You have a child and everything in the world is supposed to be right. Then, you blink, and you're standing in a children's hospital with doctors and nurses, your child hooked up to machines, and you start your journey of being in constant survival mode. You find yourself emotionally and mentally exhausted caring for a chronically sick child, whose outcome is unknown. Then you learn the news of needing an organ transplant, and it is heart wrenching. Going through this journey made me realize just how important pediatric organ donation truly is. You are not aware of all of the facts like donors needing to be appropriately matched by size, or aware that the supply of deceased donor organs for children is so small, leaving children more vulnerable to dying on the waitlist-more than adults. Because of this, advocating for pediatric transplantation has become a passion of mine. I have been able to take my own experiences as a parent, my son's experiences as a transplant recipient, and help others who are currently going through the same challenges. I have been able to help educate others the importance of organ donation and how it gives children a second chance at life. Children need a voice, and as parents, we need to be that voice for them, especially when the need for organs outweigh the availability to those children who are waiting for that life-saving gift. I have met so many amazing families and their children and have learned so much by working alongside some of the most amazing physicians and other advocates in our community.

In March of 2019, myself along with other parent advocates, physicians, transplant organizations, and non-profit organizations in the transplant community decided to collaborate together to write an open letter to the community regarding "Pediatric priority of liver transplantation." What sparked this letter of advocacy was the new liver allocation policy that was to take effect. This policy would not only benefit the sickest adult patients but also benefit pediatric patients as a whole on the waiting list. Prior to this new policy, pediatric livers were being offered to adults locally first, rather than the more critically ill children nationally. Days later, the new policy was ordered by the courts to revert back to the old policy. We wanted to make sure the pediatric voice was heard and that children received priority in the matter. As of today, the letter has reached close to 15,000 signatures on https://www.change.org and we as a community are still advocating and pushing for pediatric priority in liver transplantation.

As previously mentioned, Nathan takes immune suppression medication to keep his liver healthy. It would be nice to see more research and education on the long-term effects of immune suppression medications. From the very beginning of transplant, you are always warned that sometimes you end up trading one disease for another. Although not every patient is a victim of the

${ }^{1}$ Montgomery, IL, USA
Correspondence: Jen Lau (info@pedres.org)

Received: 7 November 2019 Accepted: 7 November 2019

Published online: 14 November 2019 
effects of these medications, Nathan posttransplant has developed severe allergic diseases, including food allergies, asthma, and eosinophilic esophagitis. More studies and education devoted to these "common" posttransplant diseases could help with a higher understanding on what exactly the role of immune suppression medications may have and could help develop better treatment options for patients who have unfortunately experienced the effects. It may also lead to a better immune suppression medication that may have less long-term effects in general.

Organ transplantation has come a very long way. From cadaveric to living donations, split liver to domino kidney transplants, these are all advances in transplantation, but there are still issues the community faces. I think the biggest issue in pediatric organ transplant, like in adult transplant, is merely not having enough organs. How do we get more donors? We advocate, educate, and continue to push the envelope in the transplant community. Maybe instead of an opt-in system where we have to actively sign up to be an organ donor, we move to an opt-out system where everyone becomes a donor like other countries? In the liver transplant arena, we could educate and train more surgeons and transplant centers to perform split liver transplants, where one deceased donor organ is used for two recipients (typically a child and an adult). Researchers should continue to work on regenerative medicine and three-dimensional printing, so one day we do not have to worry about the scarcity of organs.

As a parent of a liver transplant recipient, I give my gratitude to the physicians, surgeons, and transplant community for advocating for children who sit and wait for that lifesaving gift and for their parents who worry that gift will never come. It has been amazing to see the pediatric transplant community recently come together and advocate together. I believe it takes a village to advocate in this community, and if we keep collaborating and maintaining a team-like approach in pediatric transplant medicine, the more voices will be heard and more positive changes for our children will happen. 